\title{
Hybrid versus Norwood procedure for hypoplastic left heart syndrome: Contemporary series from a single center
}

\author{
Alexander A. Brescia, MS, ${ }^{a}$ Saadeh Jureidini, MD, ${ }^{b}$ Saar Danon, MD, ${ }^{\mathrm{b}}$ Eric Armbrecht, PhD, \\ Andrew C. Fiore, MD, ${ }^{a}$ and Charles B. Huddleston, MD $^{a}$
}

\begin{abstract}
Objective: Two different strategies have emerged in the initial palliation for hypoplastic left heart syndrome, the conventional Norwood operation and the so-called hybrid procedure. We have used each of these at our center. The purpose of the present study was to compare the outcomes of both procedures.
\end{abstract}

Methods: From 2007 to 2012, 40 patients presented to the Cardinal Glennon Children's Medical Center with hypoplastic left heart syndrome or 1 of its variants. Of the 40 patients, 24 underwent a hybrid procedure and 16 a Norwood procedure for initial palliation. The medical records, echocardiograms, and cardiac catheterization data were retrospectively reviewed. Standard statistical analysis was performed.

\begin{abstract}
Results: The patients who underwent the hybrid procedure weighed less than those who underwent the Norwood procedure. Overall unadjusted survival was better in the Norwood group, although this did not reach statistical significance. Overall hospital resource usage was similar in both cohorts, taking into account both first and second palliation stages.
\end{abstract}

Conclusions: In our review, we found no statistically significant difference in survival or resource usage between those patients undergoing the Norwood procedure and those undergoing a hybrid procedure as initial palliation for hypoplastic left heart syndrome. (J Thorac Cardiovasc Surg 2014;147:1777-82)

The palliation for hypoplastic left heart syndrome (HLHS) has evolved into 2 staged surgical strategies to achieve an unobstructed systemic outflow tract, unrestricted intraatrial communication, a controlled source of pulmonary blood flow, and a reliable source of coronary blood flow. Currently, the Norwood operation can achieve these goals, with a 30 -day mortality of $11.5 \%$ in average risk patients. ${ }^{1}$ However, neonates with associated prematurity, an intact atrial septum, severe valve insufficiency, a low birth weight $(<2.5 \mathrm{~kg})$, noncardiac conditions, or genetic abnormalities are considered high-risk candidates with a reported stage I hospital mortality of $40 \%$ to $60 \%{ }^{2}$

For these high-risk patients with HLHS, an alternative hybrid strategy was developed, consisting of stenting of the ductus arteriosus and banding the branch pulmonary arteries, with encouraging early results. ${ }^{3}$ This procedure avoids neonatal cardiopulmonary bypass and arch

\footnotetext{
From the Divisions of Cardiothoracic Surgery, ${ }^{\mathrm{a}}$ Pediatric Cardiology, ${ }^{\mathrm{b}}$ and Pediatrics, ${ }^{\mathrm{c}}$ St Louis University School of Medicine and Cardinal Glennon Medical Center, St Louis, Mo.

Mr Brescia was supported by the American Association for Thoracic Surgery Summer Intern Scholarship.

Disclosures: Authors have nothing to disclose with regard to commercial support.

Read at the 39th Annual Meeting of The Western Thoracic Surgical Association, Coeur d'Alene, Idaho, June 26-29, 2013.

Received for publication July 3, 2013; revisions received Feb 15, 2014; accepted for publication Feb 20, 2014; available ahead of print March 28, 2014.

Address for reprints: Charles B. Huddleston, MD, Division of Cardiothoracic Surgery, Cardinal Glennon Medical Center, 1465 S Grand Blvd, Ste 532, St Louis, MO 63104 (E-mail: chuddle7@slu.edu).

$0022-5223 / \$ 36.00$

Copyright (c) 2014 by The American Association for Thoracic Surgery

http://dx.doi.org/10.1016/j.jtcvs.2014.02.066
}

reconstruction, deferring these procedures to stage II palliation (SIIP) at 4 to 6 months of age when such high-risk patients theoretically can tolerate a longer and more complex operation. Some centers have adopted this strategy for initial palliation in all patients with HLHS. However, important potential disadvantages of the hybrid technique at the first stage include retrograde arch obstruction from the ductal stent, resulting in coronary and cerebral malperfusion; restrictive intra-atrial communication, resulting in early and late pulmonary venous obstruction; and early and late mechanical distortion of the branch pulmonary arteries, resulting in an imbalance of systemic and pulmonary blood flow and injury to the branch pulmonary arteries. Each of these disadvantages can profoundly inhibit the achievement of a total cavopulmonary connection and long-term survival.

The purpose of the present report was to retrospectively compare our results with both strategies, including our learning curve, with an emphasis on unadjusted early and midterm survival, hemodynamics, pulmonary artery (PA) growth, morbidity, and time-related resource usage.

\section{METHODS}

\section{Patients}

We conducted a retrospective review of 40 consecutive patients with HLHS or 1 of its variants who had undergone staged single ventricle palliation with either a hybrid $(\mathrm{n}=24)$ or Norwood $(\mathrm{n}=16)$ strategy from January 2007 to December 2012. The institutional review boards of St Louis University and Cardinal Glennon Children's Medical Center approved the study and waived the need for patient consent. The analysis excluded any patients enrolled in either an interim strategy for heart 


\section{Abbreviations and Acronyms \\ HLHS = hypoplastic left heart syndrome \\ SIP $=$ stage I palliation \\ SIIP $=$ stage II palliation}

transplantation or a bridge to 2-ventricle repair. No patient in the Norwood group underwent an emergent salvage operation.

The decision regarding initial surgical palliation varied during the study period. For the first 1.5 years of the study, all patients, without exception, underwent a Norwood procedure. For the subsequent 2 years, all patients, without exception, underwent a hybrid procedure. In the final 1.5 years of the study, the pathway of surgical palliation was decided during an interdisciplinary conference attended by representatives from cardiology, cardiac surgery, and critical care on an individual patient basis.

The demographic information, cardiac anatomy, hemodynamics and echocardiographic variables, and all postoperative outcomes were recorded retrospectively from the patient medical records. The families, cardiologists, and primary care pediatricians were interviewed as necessary to gather complete follow-up information.

Echocardiography was performed at admission, before discharge after stage I palliation (SIP) and before SIIP, before discharge after SIIP, and at the latest follow-up examination. Pulmonary insufficiency was graded according to established criteria as follows: trace to mild, 0 ; mild, 1; moderate, 2; severe, $3{ }^{4}$ Cardiac catheterization was performed to assess the hemodynamic variables before SIIP in all patients.

The follow-up data were complete for 26 hospital survivors and not significantly different between the 2 cohorts (hybrid group, $741 \pm 725$ days; Norwood group, $524 \pm 530$ days; $P<.31$ ).

Early death was defined as death in the operating room, in the hospital postoperatively, or within 30 days of surgery, regardless of discharge status. Death after discharge or $>30$ days after the procedure was considered late. Interstage death was defined as death occurring after discharge from SIP hospitalization and before SIIP.

The day of surgery was the first recorded day for the hospital stay, intensive care unit stay, and intubation period. For patients not discharged between SIP and SIIP, the total interstage duration in days was used as the hospital stay after SIP. The duration of continuous intubation included patients reintubated within 24 hours of extubation. Reintervention at any time included any cardiac reoperation, diaphragm plication, a transcatheter procedure for recurrent coarctation, the development of a restrictive atrial septum, and PA or shunt stenosis. The patient demographic profile, cardiac morphology, and diagnosis are summarized in Table 1.

\section{Surgical Management}

Norwood procedure. Norwood palliation included sternotomy and right atrial and innominate artery cannulation with a polytetrafluoroethylene (Gore-Tex, W. L. Gore \& Associates, Inc, Flagstaff, Ariz) graft, with hypothermia to $18^{\circ} \mathrm{C}$. After ductal division, the distal aortic arch was augmented 5 to $10 \mathrm{~mm}$ beyond the insertion of the ductus arteriosus using a homograft patch with the heart beating and full brain perfusion during core cooling. The proximal arch reconstruction and PA to ascending aorta amalgamation (Damus-Kaye-Stansel) and atrial septectomy were performed with the heart arrested and regional cerebral perfusion at 40 to $45 \mathrm{~mL} / \mathrm{kg} / \mathrm{min}$. The modified Blalock-Taussig shunt (3 patients) or polytetrafluoroethylene (Gore-Tex, W. L. Gore \& Associates, Inc) right ventricular to PA conduit (13 patients) was inserted with the heart beating during rewarming after atrial closure, cardiac air removal, and systemic reperfusion in accordance with published techniques. The type of systemic to pulmonary shunt used was decided by the surgeon and chosen in accordance with what surgeon thought was best suited for the anatomy. Dopamine, milrinone, epinephrine, and delayed sternal closure were used postoperatively in selected patients. Phenoxybenzamine was not used in any patient.

SIIP consisted of bidirectional cavopulmonary anastomosis in all patients. Bidirectional Glenn and PA reconstruction were performed with the heart beating, and cardioplegia was used for repeat atrial septectomy or repair of the tricuspid valve. Regional cerebral perfusion through the innominate artery and cardioplegia were used when patch enlargement of the neo-aorta or coarctation site was required. A left superior vena cava was routinely anastomosed to the left PA. The patch material was either a pulmonary homograft or autologous pericardium.

\section{Hybrid Procedure}

SIP was performed according to the technique previously described by Galantowicz and colleagues. ${ }^{5}$ PA banding used a segment of 3.0- or 3.5-mm polytetrafluoroethylene (Gore-Tex, W. L. Gore \& Associates, Inc) tube wrapped around each branch PA. Surface echocardiography was used to assess the band tightness, with a goal velocity of 3 to $4 \mathrm{~m} / \mathrm{s}$. Balloon atrial septostomy was performed as a separate procedure, and atrial stents were not used. SIIP was performed in accordance with previously published techniques. ${ }^{6}$ The aortic arch augmentation after stent removal and atrial septectomy used regional cerebral perfusion, as described for the Norwood procedure. During rewarming, with the heart beating, the distal main PA was reconstructed with a homograft, the right PA was augmented at the band site with the cavopulmonary anastomosis, and the left PA was balloon dilated intraoperatively.

Modified ultrafiltration was used in all patients after each operation. No patient in either cohort crossed over. Our home interstage monitoring program, as described by Ghanayem and colleagues, ${ }^{7}$ was applied equally to all patients.

\section{Statistical Analysis}

The outcomes were tabulated using an intent-to-treat analysis. Continuous data are presented as the median and range and mean \pm standard deviation, as appropriate. The categorical characteristics are given as counts and percentages and were compared using Fisher's exact test or the chi-square test, as appropriate. Two group comparisons of continuous variables and outcomes were compared using unpaired 2-tailed $t$ tests. Late outcomes are expressed in a time-dependent manner using Kaplan-Meier analysis and were compared using a log-rank test. All analyses were performed using the Statistical Package for Social Sciences (SPSS, Inc, Chicago, Ill).

\section{RESULTS \\ Patient Profile and Anatomy}

The demographic and morphologic characteristics of the 2 cohorts are summarized in Table 1. The mean age of the hybrid cohort was older, but the hybrid patients had a significantly lower weight, with a greater proportion weighing $<2.5 \mathrm{~kg}$. The frequency of aortic atresia and the morphologic subtypes of HLHS were comparable, with a nearly equal distribution of coexisting anatomic lesions.

\section{Perioperative and Interstage Data}

Stage I. At completion of SIP, the right and left band velocity was similar in the hybrid group and the discharge oxygen saturations were similar between the 2 cohorts. The overall postintervention extracorporeal membrane oxygenation requirement was low but showed a trend toward being greater in the Norwood group (Table 2).

Interstage hemodynamics. The hemodynamic evaluation before SIIP occurred at a mean of 5 months in both cohorts 
TABLE 1. Patient characteristics

\begin{tabular}{lccr}
\hline \multicolumn{1}{c}{ Characteristic } & Hybrid $(\mathbf{n = 2 4 )}$ & Norwood $(\mathbf{n}=\mathbf{1 6})$ & $\boldsymbol{P}$ value \\
\hline Demographics & & & \\
Male gender & $16(68)$ & $8(50)$ & .29 \\
Age at SIP $(\mathrm{d})$ & $10 \pm 7$ & $6 \pm 2$ & .03 \\
Weight at SIP $(\mathrm{kg})$ & $2.6 \pm 0.7$ & $3.1 \pm 0.5$ & .03 \\
Weight $<2.5 \mathrm{~kg}$ & $11(46)$ & $1(6)$ & .01 \\
Genetic syndrome & $4(17)$ & $0(0)$ & .10 \\
Morphology & & & \\
HLHS & & & \\
AA/MA & $6(25)$ & $3(19)$ & .72 \\
AA/MS & $4(17)$ & $4(25)$ & .69 \\
AS/MA & $1(4)$ & $1(6)$ & 1.00 \\
AS/MS & $9(37)$ & $3(19)$ & .69 \\
Aortic atresia & $10(42)$ & $7(44)$ & .89 \\
Unbalanced AVC & $2(8)$ & $0(0)$ & .51 \\
Double outlet RV & $2(8)$ & $0(0)$ & .51 \\
Double inlet LV & $2(8)$ & $3(19)$ & .63 \\
Coexisting anatomy & & & \\
VSD & $2(8)$ & $2(12)$ & 1.00 \\
TAPVR & $2(8)$ & $1(6)$ & 1.00 \\
Intact atrial septum & $1(4)$ & $1(3)$ & 1.00 \\
Heterotaxy & $1(4)$ & $0(0)$ & 1.00 \\
Dextrocardia & $1(4)$ & $0(0)$ & 1.00 \\
IAA & $0(0)$ & $1(6)$ & 1.00 \\
\hline Da & & & \\
\hline
\end{tabular}

Data presented as mean \pm standard deviation or $\mathrm{n}(\%)$. SIP, Stage I palliation; $H L H S$, hypoplastic left heart syndrome; $A A / M A$, aortic atresia/mitral atresia; $A A / M S$, aortic atresia/mitral stenosis; $A S / M A$, aortic stenosis/mitral atresia; $A S / M S$, aortic stenosis/ mitral stenosis; $A V C$, atrioventricular canal; $R V$, right ventricle; $L V$, left ventricle; $V S D$, ventricular septal defect; TAPVR, total anomalous pulmonary venous return; $I A A$, interrupted aortic arch.

(Table 2). The arterial oxygen saturation had decreased to approximately $70 \%$ from the SIP levels of approximately $80 \%$. We can infer that single ventricular function was well preserved in both groups because of the low enddiastolic pressure and similar superior caval oxygen saturations. In the hybrid patients, band tightness would seem to have remained adequate because of the relatively low mean distal PA pressure. However, the pulmonaryto-systemic flow ratio was significantly greater in the hybrid patients, although the PA pressures and ventricular enddiastolic pressures were nearly the same. This would suggest lower pulmonary vascular resistance in the hybrid group.

Stage II palliation. SIIP occurred at a mean of approximately 5 months of age in the hybrid and Norwood patients (Table 2). The cardiopulmonary bypass and global myocardial ischemic times were significantly greater in the hybrid group than in their Norwood counterparts, as would be expected given the differences in the operations. At the latest follow-up point, the peripheral oxygen saturation had remained similar in all patients.

\section{Reintervention}

A total of 32 reinterventions were performed in 20 patients (hybrid, 11 of $24,46 \%$; Norwood, 9 of $16,50 \%$;
TABLE 2. Perioperative data at SIP and SIIP

\begin{tabular}{|c|c|c|c|}
\hline Variable & Hybrid & Norwood & $P$ value \\
\hline \multicolumn{4}{|l|}{ SIP } \\
\hline CPB time (min) & NA & $167 \pm 54$ & \\
\hline Crossclamp time (min) & NA & $63 \pm 42$ & \\
\hline \multicolumn{4}{|l|}{ PA band velocity } \\
\hline $\mathrm{RPA}(\mathrm{m} / \mathrm{s})$ & $3.7 \pm 0.5$ & NA & \\
\hline LPA (m/s) & $3.8 \pm 0.6$ & NA & \\
\hline Discharge $\mathrm{SaO}_{2}(\%)$ & $86 \pm 4$ & $83 \pm 6$ & .16 \\
\hline ECMO & $1(4)$ & $2(13)$ & 6 \\
\hline \multicolumn{4}{|l|}{ Interstage hemodynamics } \\
\hline Age (d) & $131 \pm 43$ & $130 \pm 46$ & .93 \\
\hline Aortic saturation $(\%)$ & $71 \pm 6$ & $68 \pm 5$ & .20 \\
\hline Superior caval saturation $(\%)$ & $52 \pm 3$ & $47 \pm 8$ & .15 \\
\hline RVEDP (mm Hg) & $10 \pm 1$ & $10 \pm 3$ & .4 \\
\hline Qp/Qs & $1.3 \pm 0.08$ & $0.72 \pm 0.35$ & .03 \\
\hline Mean PA (mm Hg) & $15 \pm 6$ & $15 \pm 2$ & .75 \\
\hline \multicolumn{4}{|l|}{ SIIP } \\
\hline Age (d) & $161 \pm 32$ & $151 \pm 40$ & .44 \\
\hline CPB time $(\mathrm{min})$ & $217 \pm 33$ & $89 \pm 32$ & .001 \\
\hline Crossclamp time (min) & $116 \pm 29$ & 0 & NA \\
\hline Latest $\mathrm{SaO}_{2}(\%)$ & $81 \pm 6$ & $82 \pm 4$ & .65 \\
\hline ECMO & $0 \pm 0$ & $1(8)$ & 1.0 \\
\hline
\end{tabular}

Data presented as n (\%), median, or mean \pm standard deviation. SIP, Stage I palliation; SIIP, stage II palliation; $C P B$, cardiopulmonary bypass; $P A$, pulmonary artery; $R P A$, right pulmonary artery; $L P A$, left pulmonary artery; $\mathrm{SaO}_{2}$, oxygen saturation; $E C M O$, extracorporeal membrane oxygenation; $R V E D P$, right ventricle enddiastolic pressure; $Q p / Q s$, pulmonary blood flow/systemic blood flow ratio; $N A$, not applicable.

$P=.5$ ) during the entire study (Table 3). No patient required a separate readmission to the hospital for these interventions. Some were performed during the pre-SIIP cardiac catheterization. Of the 17 transcatheter interventions, 5 $(29 \%)$ involved stenting residual coarctation after the Norwood procedure, and 5 hybrid patients required balloon and stent intervention on the left PA (3 patients) or vein (1 patient, twice). The most frequent surgical reintervention was insertion of a modified Blalock-Taussig shunt. Two hybrid patients developed discontinuous left PA (before routine use of intraoperative balloon dilatation) and required $\mathrm{PA}$ rehabilitation with a systemic shunt. Both patients successfully underwent completion Fontan, 1 to a single lung. A hybrid patient with total anomalous pulmonary venous return required Glenn takedown at SIIP and died late of gastrointestinal bleeding. A second patient with severe tricuspid regurgitation required urgent Glenn takedown after hybrid SIIP. Late survival was accomplished with staged valve repair followed by conversion back to a Glenn shunt. The remaining Blalock-Taussig shunt was inserted into a Norwood patient with early obstruction of the Sano shunt.

\section{Resource Usage}

After SIP, resource usage was greater for the Norwood patients, shown by the significantly longer ventilation and intensive care unit times and a trend toward longer overall 
TABLE 3. Resource usage

\begin{tabular}{|c|c|c|c|c|c|c|c|c|c|}
\hline \multirow[b]{2}{*}{ Variable } & \multicolumn{3}{|c|}{ SIP } & \multicolumn{3}{|c|}{ SIIP } & \multicolumn{3}{|c|}{ SIP and SIIP } \\
\hline & Hybrid & Norwood & $P$ value & Hybrid & Norwood & $P$ value & Hybrid & Norwood & $P$ value \\
\hline Intubation time (d) & 6 & 10 & .07 & 6 & 1 & .2 & 9 & 12 & .31 \\
\hline ICU stay (d) & 10 & 16 & .01 & 16 & 5 & .001 & 23 & 21 & .9 \\
\hline Hospital stay (d) & 19 & 25 & .1 & 24 & 8 & .01 & 47 & 31 & .4 \\
\hline
\end{tabular}

hospitalization (Table 3). Comprehensive SIIP for patients enrolled in the hybrid strategy, requiring stent removal and aortic arch and PA reconstruction, not surprisingly required a longer intensive unit care and hospital stay, with a trend toward a longer ventilator time. However, the analysis of the combined resource usage for SIP plus SIIP revealed no significant overall differences in intubation time or intensive care unit and hospital stay, whether the original treatment strategy chosen was a hybrid or Norwood operation.

\section{Follow-up Evaluation}

Hybrid patients retained "band physiology" with ductal stenting, and the main PA and pulmonary valve were quite dilated. However, their pulmonary valve remained competent, and the valve annulus and sinotubular ridge diameters were not significantly different from those in the patients who underwent a Norwood strategy at late follow-up. The echocardiographically measured PA diameters were similar between the 2 cohorts, suggesting that band site reconstruction of the left PA using intraoperative balloon dilatation and of the right PA with a Glenn anastomosis is effective in the early term (Table 4).

\section{Mortality}

Mortality after SIP. Eight patients died in the hospital after SIP (hybrid, 7 of $24,29 \%$; Norwood, 1 of $16,6 \%$; $P=.1)$. The single Norwood mortality occurred from hemodynamic compromise 4 days after shunt revision. Among the hybrid cohort, 4 deaths occurred at 20, 30, 35, and 37 days secondary to necrotizing enterocolitis in 3 or liver failure in 1 . Two patients had Alagille syndrome, and one had been born with an intact atrial septum. Their mean weight was $2.0 \mathrm{~kg}$, with an adequate right and left PA band velocity of $3.5 \mathrm{~m} / \mathrm{s}$ by echocardiography. Two

TABLE 4. Latest echocardiographic assessment of pulmonary artery dimensions

\begin{tabular}{lccc}
\hline \multicolumn{1}{c}{ Variable } & Hybrid & Norwood & $\boldsymbol{P}$ value \\
\hline Pulmonary insufficiency (\%) & $0.5 \pm 0.6$ & $0.5 \pm .8$ & .9 \\
LPA diameter* & $-0.8 \pm 1.1$ & $-0.7 \pm 1.4$ & .9 \\
RPA diameter* & $-1.4 \pm 1.0$ & $-1.0 \pm 1.2$ & .3 \\
Pulmonary valve annulus diameter* & $1.5 \pm 1.0$ & $2.1 \pm 0.8$ & .1 \\
Sinotubular ridge diameter (mm) & $16 \pm 4$ & $16 \pm 2$ & .9 \\
\hline
\end{tabular}

Data presented expressed as mean \pm standard deviation. $L P A$, Left pulmonary artery; $R P A$, right pulmonary artery. *Standard deviation of z-score. additional hybrid deaths (mean weight, $1.8 \mathrm{~kg}$ ) occurred at 1 and 16 days secondary to post-transfusion hyperkalemia. The remaining hybrid mortality occurred at 85 days in a patient with trisomy 18 after support was withdrawn secondary to sepsis and the need for reintubation.

Mortality after SIIP. The 2 hospital deaths after SIIP were in the hybrid cohort (hybrid, 2 of 17, 12\%; Norwood, 0 of $15,0 \% ; P=.5)$. One patient with Pierre-Robin syndrome died on day 11 of low cardiac output and family denial for mechanical support. The remaining death occurred 27 days after SIIP secondary to aspiration pneumonia and generalized sepsis.

Late mortality. Four patients (hybrid, 2; Norwood, 2) died late postoperatively at $72,122,134$, and 773 days after SIIP secondary to gastrointestinal bleeding, ventricular fibrillation at home, viral pneumonia, and severe ventricular failure while awaiting transplantation, respectively.

The cumulative unadjusted survival (hybrid, 13 of 24, $54 \%$; Norwood, 13 of $16,81 \% ; P<.08)$ and 1 -year Kaplan-Meier actuarial survival (hybrid, 58\%; Norwood $81 \% ; P<.11)$ showed a trend toward being greater in the Norwood cohort, but the difference was not significant (Figure 1). Given the low mortality rate in the Norwood group and small sample size, a logistic regression model

One-Year Survival: Hybrid vs. Norwood

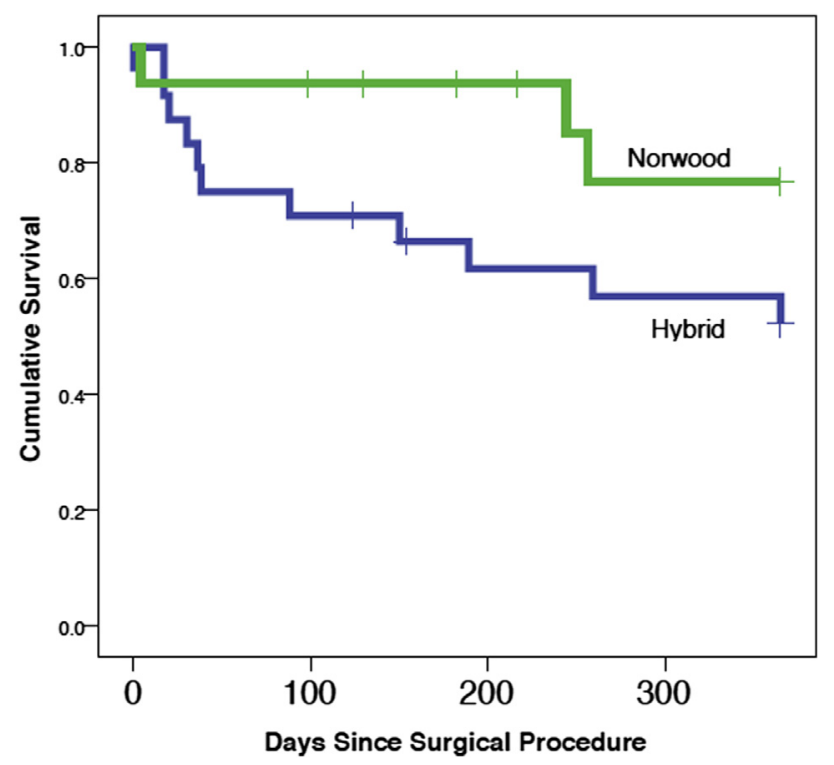

FIGURE 1. Graph showing 1-year survival stratified by procedure. 
to attempt to adjust for differences between the groups was not possible. The groups were not equivalent. We conducted a special analysis in which we matched the hybrid and Norwood patients by age. In that sample, all statistically significant differences in the demographics between the 2 groups were eliminated, and we found no differences in the cumulative survival between the 2 groups (12 of 16 hybrid, 13 of 16 Norwood; $P=1.0$ ).

\section{DISCUSSION}

The treatment of HLHS has progressed from comfort care only (with essentially $100 \%$ mortality) to aggressive reconstructive surgery (with $15 \%$ mortality) at most major pediatric medical centers during the past 25 years. The introduction of a variety of modifications in the surgical techniques and refinements in postoperative care were primarily responsible for this improvement. However, controversies remain concerning the precise nature of the initial palliation for these patients, in particular, for those deemed to be high risk. The present review of our series of patients who had undergone either the hybrid or Norwood procedure was an attempt at validating which strategy would be more appropriate for patients with HLHS.

The hybrid procedure has been touted as a safer approach because cardiopulmonary bypass, myocardial ischemia, and circulatory arrest will be avoided in the newborn. ${ }^{5}$ The hybrid procedure has been the procedure of choice in some centers but has been reserved for high-risk patients in others. ${ }^{8}$ It is not clear that the hybrid procedure is a safer approach than conventional reconstructive surgery as initial palliation in either group. ${ }^{9}$ The study by Pizarro and colleagues ${ }^{9}$ found no differences in the survival of patients undergoing the hybrid procedure compared with those undergoing the conventional Norwood operation. Theirs was not a randomized study, but all patients were deemed "high risk" on the basis of the preoperative characteristics and were similar in most parameters. Venugopal and colleagues $^{8}$ reported on their experience with the hybrid procedure for 21 patients deemed at high risk for the Norwood operation. They had an overall mortality of $38 \%$. This was similar to the mortality reported for the Norwood operation in high-risk patients at 2 different centers. ${ }^{10,11}$ Thus, regarding the survival benefit, it remains unclear whether the hybrid procedure has an advantage compared with the conventional Norwood procedure.

The mortality from our series was significantly greater in the hybrid group. However, the hybrid patients as a group had greater risk (Table 1). Admittedly, all deaths occurred in patients considered high risk using previously defined risk factors, primarily low birth weight. The cause of death for most of these patients appeared to be related to low systemic cardiac output owing to persistent pulmonary overcirculation, presumably the underlying cause of necrotizing enterocolitis and liver failure seen in our patients.
Necrotizing enterocolitis has been more common after the hybrid procedure than after the Norwood operation. ${ }^{12,13}$ Again, it has been presumed that this results from the relatively lower systemic cardiac output in the hybrid group than in the Norwood group. In our series, the pulmonary to systemic blood flow was significantly greater in the hybrid patients at pre-SIIP cardiac catheterization. Whether this played a role in the development of necrotizing enterocolitis in our patients at a point much earlier in their course is hypothetical. However, we are concerned about this possibility. This speaks to the difficulty in adjusting the PA bands in small infants in the hybrid catheterization laboratory, where a consistently steady state is not always achievable. It is also consistent with the findings that the early postoperative hemodynamic profile of the hybrid patients was not significantly improved compared with that seen with patients undergoing the Norwood procedure. $^{14}$

The reintervention rate in those patients undergoing the hybrid procedure was relatively high. In our series, the percentage of patients requiring reintervention was similar in each group. After the hybrid procedure, the most common reintervention has been for a restrictive atrial septum in previous studies. ${ }^{3,15}$ However, this was not seen in our series. We had a policy of delaying the initial intervention on the atrial septum, as suggested by Galantowicz and colleagues. ${ }^{5}$ We included only those patients requiring this intervention beyond the first atrial septostomy, regardless of when it was performed. The most common reintervention in the Norwood group was for repeat coarctation, occurring in $31 \%$. This is a typical incidence according to previously reported series. ${ }^{16,17}$ All the stents used have the ability for postdilation to adult size. This can be done in 2 to 3 future catheterizations, 1 of which could be at the pre-Fontan evaluation.

Regardless of the initial palliation, both groups of patients required significant resources during the first year of life. The intensive care unit stay was significantly shorter in the hybrid group after SIP but much longer after SIIP. The differences overall, for both stages, were not significant.

It can be somewhat difficult to accurately measure the PA size using echocardiography. Nonetheless, we found little difference between the 2 cohorts in terms of the diameter of each branch PA. The pulmonary valve annulus size also was not significantly different between the 2 groups. We had concerns about the enlargement of the pulmonary valve annulus and the main PA at SIIP in the hybrid group. In our early follow-up period, additional significant dilatation did not occur. Likewise, the incidence of pulmonary valve insufficiency was not different between the 2 cohorts.

The limitations in the present analysis included nonrandomized, retrospective data acquisition, no defined prospective institutional protocol to select patients for either treatment strategy, and the effect of a learning curve on 
the early and late outcomes. A post hoc power analysis indicated our study had a $45 \%$ power to detect the observed differences in SIP mortality. The sample provided a $79 \%$ power to detect twofold differences in the mean length of stay between the 2 groups; however, the observed difference was only $50 \%$ greater in the Norwood group. Although the method and management protocols were standardized, it is possible that individual differences among the physicians and surgeons could have influenced the patient outcomes. When we embarked on the present review, we believed that the 2 groups of patients would be similar because of the early pattern of strategy used (consistent Norwood procedure in the initial phase and consistent hybrid procedure in the middle phase). However, this did not prove true when the latest group of patients was added, in which smaller infants were more consistently referred for the hybrid procedure. Obviously, this would complicate any conclusions that might be drawn from our retrospective review.

This brings us back to the original question. Which of these procedures can provide the best initial palliation for HLHS? Although we found a trend for the hybrid procedure to result in greater mortality than the Norwood procedure, the 2 cohorts were not equal in risk, and a definitive answer cannot be derived from our findings. Even the high-risk group had somewhat poor outcomes that were similar to what has been reported in published studies for the Norwood procedure in high-risk patients. ${ }^{9}$ The most recent report from the Congenital Heart Surgery Society of Thoracic Surgeons Database was also somewhat sobering regarding survival after the hybrid procedure for HLHS-26.5\% mortality at SIP and $19.8 \%$ at SIIP. No details were available regarding patient selection. Likely, some patients would benefit from this approach, such as those listed for transplantation, those with a borderline left ventricle and aortic stenosis, and those with a left ventricular tumor causing inflow and outflow obstruction, with reason to believe that the tumor will regress. Because of the potential problems with stent removal at more definitive therapy, some have advocated banding the PAs and continuing to treat these patients with prostaglandin $E_{1}$. This strategy adds yet another facet to the options available for patients with HLHS. Much like the study comparing the "Sano" shunt and the Blalock-Taussig shunt for the Norwood procedure, a randomized trial controlling for many of the variables encountered with these patients will be required to adequately answer the question regarding which approach for initial palliation will be most efficacious.

\section{References}

1. Tabbutt S, Ghanayem N, Ravishankar C, Sleeper LA, Cooper DS, Frank DU, et al. Risk factors for hospital morbidity and mortality after the Norwood procedure: a report from the Pediatric Heart Network Single Ventricle Reconstruction trial. J Thorac Cardiovasc Surg. 2012;144:882-95.

2. Tabbutt S, Dominguez TE, Ravishankar C, Marino BS, Gruber PJ, Wernovsky G, et al. Outcomes after the stage I reconstruction comparing the right ventricular to pulmonary artery conduit with the modified Blalock Taussig shunt. Ann Thorac Surg. 2005;80:1582-90.

3. Bacha EA, Daves S, Hardin J, Abdulla RI, Anderson J, Kahana M, et al. Singleventricle palliation for high-risk neonates: the emergence of an alternative hybrid stage I strategy. J Thorac Cardiovasc Surg. 2006;131:163-71.

4. Zoghbi WA, Enriquez-Sarano M, Foster E, Grayburn PA, Kraft CD, Levine RA, et al. Recommendations for evaluation of the severity of native valvular regurgitation with two-dimensional and Doppler echocardiography. J Am Soc Echocardiogr. 2003;16:777-802

5. Galantowicz M, Cheatham JP, Phillips A, Cua CL, Hoffman TM, Hill SL, et al. Hybrid approach for hypoplastic left heart syndrome: intermediate results after the learning curve. Ann Thorac Surg. 2008;85:1063-71.

6. Pizarro C, Murdison KA, Derby CD, Radtke W. Stage II reconstruction after hybrid palliation for high-risk patients with a single ventricle. Ann Thorac Surg. 2008;85:1382-8.

7. Ghanayem NS, Hoffman GM, Mussatto KA, Cava JR, Frommelt PC, Rudd NA, et al. Home surveillance program prevents interstage mortality after the Norwood procedure. J Thorac Cardiovasc Surg. 2003;126:1367-77.

8. Venugopal PS, Luna KP, Anderson DR, Austin CB, Rosenthal E, Krasemann T, et al. Hybrid procedure as an alternative to surgical palliation of high-risk infants with hypoplastic left heart syndrome and its variants. J Thorac Cardiovasc Surg. 2010;139:1211-5.

9. Pizarro C, Derby CD, Baffra JM, Murdison KA, Radtke WA. Improving the outcome of high-risk neonates with hypoplastic left heart syndrome: hybrid procedure or conventional surgical palliation? Eur J Cardiothorac Surg. 2008;131: 412-7.

10. Stasik CN, Gelehrter S, Goldberg CS, Bove EL, Devaney EJ, Ohve RG. Current outcomes and risk factors for the Norwood procedure. J Thorac Cardiovas Surg. 2006;131:412-7.

11. Photiadis J, Sinzobahamvya N, Haun C, Schneider M, Zartner P, Schindler E, et al. Does the shunt type determine mid-term outcome after Norwood operation? Eur J Cardiothorac Surg. 2012;42:209-15.

12. Weiss SL, Gossett JG, Kaushal S, Wang D, Backer CL, Wald EL. Comparison of gastrointestinal morbidity after Norwood and hybrid palliation for complex heart defects. Pediatr Cardiol. 2011;32:391-8.

13. Luce WA, Schwartz RM, Beauseau W, Giannone PJ, Boettner BL, Cheatham JP, et al. Necrotizing enterocolitis in neonates undergoing the hybrid approach to complex congenital heart disease. Pediatr Crit Care Med. 2011;12:46-51.

14. Li J, Zhang G, Benson L, Holtby H, Cai S, Humpl T, et al. Comparison of the profiles of postoperative systemic hemodynamics and oxygen transport in neonates after the hybrid or the Norwood procedure: a pilot study. Circulation. 2007;116(Suppl II):179-87.

15. Pilla CB, Pedra CA, Nogueira AJ, Jatene M, Souza LC, Pedra SR, et al. Hybrid management for hypoplastic left heart syndrome: an experience from Brazil. Pediatr Cardiol. 2008;29:498-506.

16. Hansen JH, Runge U, Uebing A, Scheewe J, Kramer HH, Fischer G. Cardiac catheterization and interventional procedures as part of staged surgical palliation for hypoplastic left heart syndrome. Congenit Heart Dis. 2012;7:565-74.

17. Sakurai T, Rogers V, Stickley J, Khan N, Jones TJ, Barron DJ, et al. Single-center experience of arch reconstruction in the setting of Norwood operation. Ann Thorac Surg. 2012;94:1534-9. 